\title{
EphrinB2 signalling modulates the neural differentiation of human dental pulp stem cells
}

\author{
BOON CHIN HENG ${ }^{1-3}$, TING GONG ${ }^{1,2}$, JIANGUANG XU ${ }^{1,2}$, LEE WEI LIM $^{3,4}$ and CHENGFEI ZHANG ${ }^{1,2}$ \\ ${ }^{1}$ Discipline of Endodontology, Faculty of Dentistry, The Prince Philip Dental Hospital, The University of \\ Hong Kong, Hong Kong, SAR; ${ }^{2}$ The University of Hong Kong Shenzhen Institute of Research and Innovation, \\ Shenzhen, Guangdong 518057, P.R. China; ${ }^{3}$ Faculty of Science and Technology, Sunway University, \\ Selangor Darul Ehsan 47500, Malaysia; ${ }^{4}$ School of Biomedical Sciences, Li Ka Shing Faculty of \\ Medicine, The University of Hong Kong, Hong Kong, SAR, P.R. China
}

Received February 6, 2018; Accepted May 4, 2018

DOI: $10.3892 /$ br.2018.1108

\begin{abstract}
Dental pulp stem cells (DPSCs) originate from the embryonic neural crest and have neurogenic potential. The present study investigated the roles of the forward and reverse EphrinB2 signalling pathways during DPSC neurogenesis. Treatment of DPSCs with recombinant EphrinB2-Fc protein over 7 days in a neural induction culture resulted in significant downregulation of the following neural markers: $\beta$ III-Tubulin, neural cell adhesion molecule (NCAM), nestin, neurogenin 2 (NGN2), neurofilament medium polypeptide and Musashil. Immunocytochemistry revealed that EphrinB2-Fc-treated DPSCs exhibited more rounded morphologies with fewer neurite outgrowths as well as reduced protein expression of $\beta$ III-tubulin and NGN2. Treatment of DPSCs with a peptide inhibitor specific to the EphB4 receptor significantly upregulated expression of the neural markers microtubule-associated protein 2, Musashi1, NGN2 and neuron-specific enolase, whereas treatment with a peptide inhibitor specific to the EphB2 receptor exerted negligible effects on neurogenesis. Transgenic expression of EphrinB2 in DPSCs resulted in significant upregulation of Musashi1 and NCAM gene expression, while treatment of DPSCs with recombinant EphB4-Fc protein led to significant upregulation of only Musashi1. Thus, it may be concluded that stimulation of forward EphrinB2-EphB4 signalling markedly inhibited neurogenesis in DPSCs, whereas suppression of this forward signalling pathway with peptide inhibitor specific to EphB4 promoted neurogenesis. Meanwhile, stimulation of reverse EphB4-EphrinB2 signalling only marginally
\end{abstract}

Correspondence to: Professor Chengfei Zhang, Discipline of Endodontology, Faculty of Dentistry, The Prince Philip Dental Hospital, The University of Hong Kong, 34 Hospital Road, Sai Ying Pun, Hong Kong, SAR, P.R. China

E-mail: zhangcf@hku.hk

Key words: dental pulp, differentiation, EphrinB2, neurogenesis, stem cells enhanced the neural differentiation of DPSCs. The present findings indicate the potential application of peptide or small molecule inhibitors of EphrinB2 forward signalling in neural tissue engineering with DPSCs.

\section{Introduction}

Since human dental pulp stem cells (DPSCs) were first isolated by Gronthos et al $(1,2)$, these cells have been widely utilized in regenerative medicine and tissue engineering applications $(3,4)$, mainly due to their multilineage differentiation plasticity and high proliferative capacity (5). Of particular interest are the potential therapeutic applications of DPSCs in various neurodegenerative diseases and neural disorders, due to the high innate propensity of these cells to differentiate into the neural lineage (6) as a consequence of their origin from the embryonic neural crest (7)

To date, transplanted DPSCs have demonstrated promise in the treatment of various neural lesions in animal models of stroke (8), spinal cord injury (9), facial nerve injury (10) and optical nerve injury (11), among others. Nevertheless, it must be noted that these animal studies utilized undifferentiated DPSCs, and it is probable that some degree of neural induction or neural lineage commitment of DPSCs in vitro, prior to transplantation in vivo, could further enhance the neuroregenerative potential of these adult stem cells (6). This may be facilitated by an improved understanding of the mechanisms of neurogenesis of DPSCs.

The signalling pathways mediated by the family of EphB receptors and EphrinB ligands, which are expressed in the perivascular niche of the dental pulp, are known to serve key roles in the proliferation, mobilization and mineralization of DPSCs during the process of tooth regeneration, in response to injury of the dentin matrix (12). Of particular interest in the field of neuroregeneration are EphrinB2 and its cognate receptors EphB2 and EphB4, which have been demonstrated to serve key developmental roles in embryonic neurogenesis (13-15) as well as in the homeostasis and mobilization of neural stem cells (16) and neural progenitors (17). EphrinB2 and its cognate receptors EphB2 and EphB4 are transmembrane receptor tyrosine kinases, which are established to play crucial 
roles in cell migration and in the determination of cell lineage fate (18). Simultaneous bidirectional signals are generated by the binding interactions of EphrinB2 with its cognate receptors EphB2 and EphB4, with forward signalling being effected through EphB2 and EphB4 and reverse signalling being effected through EphrinB2 (19). Direct intercellular contact is required to initiate these signalling pathways, as EphrinB2, EphB2 and EphB4 are all membrane-bound protein receptors. In a previous study by our group, it was demonstrated that the EphrinB2 signalling pathway served a key role in the osteogenic/odontogenic differentiation of DPSCs (20).

At present, the role of EphrinB2 signalling in the neurogenesis of DPSCs is not clear. Therefore, the present study aimed to investigate and delineate the roles of EphrinB2 forward and reverse signalling in the neurogenesis of DPSCs in vitro. Exogenous recombinant EphrinB2-Fc and EphB4-Fc were utilized together with specific peptide inhibitors of EphB2 and EphB4 signalling (SNEW and TNYL-RAW, respectively) (21) as well as transgenic DPSCs overexpressing the EphrinB2 ligand. The peptide inhibitors SNEW and TNYL-RAW were previously demonstrated by Chrencik et al (21) to bind specifically to the EphB2 and EphB4 receptors respectively, and block the interaction of these receptors with the EphrinB2 ligand. Both forward and reverse signalling, transduced either by EphrinB2-EphB4 interaction (TNYL-RAW) or EphrinB2-EphB2 interaction (SNEW), would thus be inhibited. In the case of transgenic expression of EphrinB2, this may stimulate both forward and reverse signalling in DPSCs.

\section{Materials and methods}

Chemical reagents, culture media and supplements. Unless otherwise stated, all chemical reagents were obtained from Sigma-Aldrich (Merck KGaA, Darmstadt, Germany), all culture media and supplements were obtained from Thermo Fisher Scientific, Inc. (Waltham, MA, USA), and all plastic labware consumables were obtained from BD Biosciences (Franklin Lakes, NJ, USA).

Isolation, characterization and culture of DPSCs. The DPSCs utilized in the current study were isolated from the extracted third molars of young adult patients below 25 years of age according to previously described procedure (22). The isolated DPSCs were characterized by flow cytometric analysis on the expressions of cluster of differentiation (CD)45, CD73, CD90 and CD105 as well as by multilineage differentiation assays (osteogenic, adipogenic and neurogenic induction), as previously described (22). The DPSCs were cultured in T75 culture flasks with $\alpha$-minimum essential medium supplemented with $10 \%(\mathrm{v} / \mathrm{v})$ foetal bovine serum (FBS) and $1 \%(\mathrm{v} / \mathrm{v})$ penicillin-streptomycin antibiotic solution at $37^{\circ} \mathrm{C}$ in a humidified $5 \% \mathrm{CO}_{2}$ incubator. The culture medium was refreshed every 3 to 4 days, and confluent monolayers were dissociated with $0.5 \%(\mathrm{w} / \mathrm{v})$ trypsin-EDTA for serial passage.

Transfection of DPSCs. Recombinant retroviral construct Rsv-NFB2-GFP-Bsd [human EphrinB2 gene (EFNB2) transgenic lentivirus with green fluorescent protein-blasticidin dual marker) and the corresponding empty vector Rsv-Null-Control-GFP-Bsd were obtained from Gentarget, Inc.
(San Diego, CA, USA). Monolayers of DPSCs at 50\% confluence were transduced with either the Rsv-NFB2-GFP-Bsd vector for transgenic expression of EphrinB2 gene or the empty vector (Rsv-Null-Control-GFP-Bsd) at a multiplicity of infection of 50 for a total duration of $24 \mathrm{~h}$ within a humidified $5 \% \mathrm{CO}_{2}$ incubator at $37^{\circ} \mathrm{C}$. Fresh culture medium was then applied, and stably transduced cells were selected with the addition of $2 \mu \mathrm{g} / \mathrm{ml}$ Blasticidin (EMD Millipore, Billerica, MA, USA) to the culture medium. Transduction efficiency was assessed qualitatively under fluorescence microscopy (Olympus Corporation, Tokyo, Japan).

Neural induction of DPSCs. DPSCs seeded on 6-well culture plates at a density of $2.5 \times 10^{4}$ cells $/ \mathrm{cm}^{2}$ and were cultured in neural induction medium composed of Neurobasal-A medium (cat. no. 10888-022; Thermo Fisher Scientific, Inc.) supplemented with $2 \%$ (v/v) B27 supplement (cat. no. 17504-044; Thermo Fisher Scientific, Inc.), $1 \%$ (v/v) penicillin-streptomycin antibiotic solution, $20 \mathrm{ng} / \mathrm{ml}$ epidermal growth factor (cat. no. PHG0314; Thermo Fisher Scientific, Inc.) and 40 ng/ml basic fibroblast growth factor (cat. no. PHG0024; Thermo Fisher Scientific, Inc.) (23). Depending on the particular experiment, the neural induction medium was additionally supplemented with either $2 \mu \mathrm{g} / \mathrm{ml}$ EphrinB2-Fc (cat. no. 7397-EB-05C; R\&D Systems, Minneapolis, MN, USA), $100 \mu \mathrm{M}$ of specific peptide inhibitor for EphB4 or EphB2 (TNYL-RAW or SNEW, respectively), $100 \mu \mathrm{M}$ of the corresponding control scrambled peptides (SCR-WTL or SCR-EPQ, respectively), or $2 \mu \mathrm{g} / \mathrm{ml}$ EphB4-Fc (cat. no. 446-B4-200; R\&D Systems). The TNYL-RAW peptide (YNYLFSPNGPIARAW), SNEW peptide (SNEWIQPRLPQH), and corresponding control scrambled peptides SCR-EPQ (EPQNHSWPIRQL) and SCR-WTL (WTLAIFARNYNGPSP), respectively (21), were custom-synthesized by Thermo Fisher Scientific, Inc. The EphrinB2-Fc and EphB4-Fc were clustered with goat anti-human Fc antibody (cat. no. H10000; Thermo Fisher Scientific, Inc.) at a molar ratio of $1: 5$ for $30 \mathrm{~min}$ at $37^{\circ} \mathrm{C}$ prior to being added into the culture medium (24). It is established that the EphrinB2 ligand naturally occurs as a multimer in its native membrane-bound form, and that monomeric soluble EphrinB2 ligand is unable to elicit forward signalling upon binding to either the EphB2 or EphB4 receptor (24). As such, clustering of the soluble recombinant EphrinB2-Fc with anti-Fc antibodies is required to mimic its naturally-occurring multimeric membrane-bound state, to in turn enable transduction of forward signalling (24). Neural induction was performed for a duration of 7 days at $37^{\circ} \mathrm{C}$ within a humidified $5 \% \mathrm{CO}_{2}$ incubator, after which the samples were collected for either reverse transcription-quantitative polymerase chain reaction (RT-qPCR) analysis or immunocytochemistry.

RT-qPCR analysis of neural marker expression by DPSCs. Total RNA of the cultured DPSCs was extracted with an RNeasy Plus Mini kit (Qiagen, Inc., Valencia, CA, USA) and subjected to RT with a SuperScript VILO Master Mix (Thermo Fisher Scientific, Inc.). RT-qPCR analysis was performed with a StepOne Real-Time PCR system (Applied Biosystems; Thermo Fisher Scientific, Inc.) utilizing SYBR Premix Ex Taq II (cat. no. RR820A; Takara Bio, Inc., Otsu, Japan). The primer sequences of neural markers [ $\beta$ III-tubulin, 

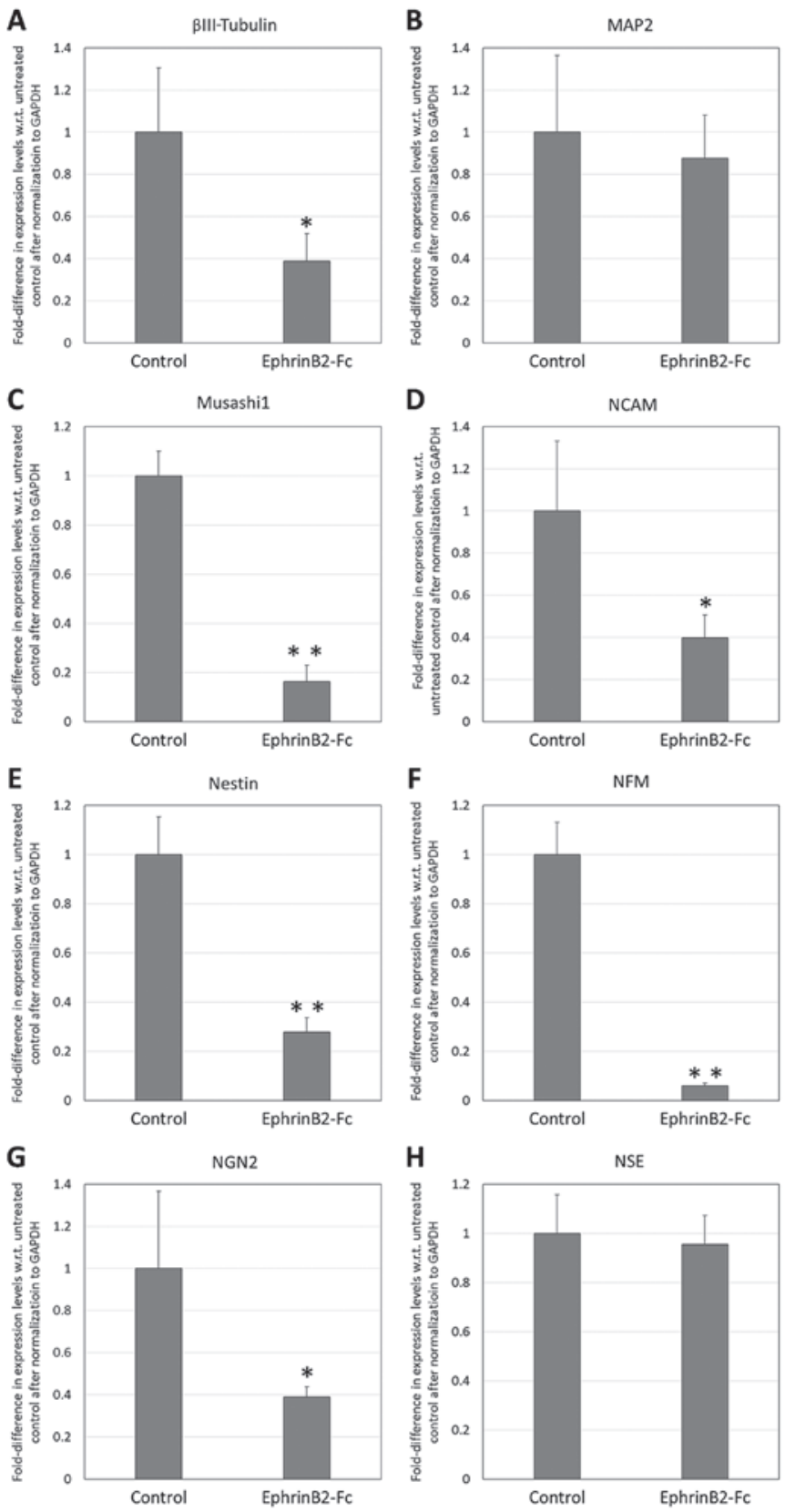

Figure 1. Reverse transcription-quantitative polymerase chain reaction analysis of neural marker expression by dental pulp stem cells following treatment with $2 \mu \mathrm{g} / \mathrm{ml}$ EphrinB2-Fc in neural induction medium for 7 days. Fold-difference in expression with respect to untreated controls is shown for (A) BIII-tubulin, (B) MAP2, (C) Musashi1, (D) NCAM, (E) nestin, (F) NFM, (G) NGN2 and (H) NSE. ${ }^{*} \mathrm{P}<0.05$ and ${ }^{* *} \mathrm{P}<0.01$ vs. Control. MAP2, microtubule-associated protein 2; NCAM, neural cell adhesion molecule; NFM, neurofilament medium polypeptide; NGN2, neurogenin 2; NSE, neuron-specific enolase.

microtubule-associated protein 2 (MAP2), Musashi1, neural cell adhesion molecule (NCAM), nestin, neurofilament medium polypeptide (NFM), neurogenin 2 (NGN2) and neuron-specific enolase (NSE)] utilized for the RT-qPCR analysis were identical to those of our previous study (23), and GAPDH was used as the endogenous reference control gene. The primer sequences used for EphrinB2, EphB2, EphB4 and GAPDH were as follows: EphrinB2 forward, 5'-CCTCTCCTC AACTGTGCCAAA-3' and reverse, 5'-CCCAGAGGTTAG GGCTGAATT-3'; EphB2 forward, 5'-ATGAACACGATC
CGCACGTA-3' and reverse, 5'-TTGGTCCGTAGCCAGTTG TTCT-3'; EphB4 forward, 5'-CCTTCCTGCGGCTAA ACGAC-3' and reverse, 5'-GTTGACTAGGATGTTGCGAG-3'; and GAPDH forward, 5'-GGCATGGACTGTGGTCATGAG-3' and reverse, 5'-TGCACCACCAACTGCTTAGC-3'. The following amplification parameters were utilized for the RT-qPCR analysis: $2 \mathrm{~min}$ at $50^{\circ} \mathrm{C}, 20 \mathrm{sec}$ at $95^{\circ} \mathrm{C}$, and 40 cycles of $3 \mathrm{sec}$ at $95^{\circ} \mathrm{C}$ followed by $30 \mathrm{sec}$ at $60^{\circ} \mathrm{C}$. The $2^{-\Delta \Delta \mathrm{Cq}}$ method (25) was used to compute the relative cycle threshold (Cq) values for each gene, which were then normalized against the endogenous gene expression of GAPDH. The fold-differences in gene expression by the DPSCs that were subjected to neural induction in the various experimental groups were normalized with respect to the DPSCs in the corresponding control groups on day 7. Overall, three experimental replicates were performed for each gene analysed by RT-qPCR.

Immunocytochemistry for the detection of $\beta I I I$-tubulin and NGN2 expression by DPSCs. After 7 days of neural induction in the presence or absence of $2 \mu \mathrm{g} / \mathrm{ml}$ EphrinB2-Fc, the DPSCs were fixed at room temperature with $4 \%(\mathrm{v} / \mathrm{v})$ paraformaldehyde for $20 \mathrm{~min}$, followed by permeabilization with $0.1 \%$ (w/v) Triton X-100 for $10 \mathrm{~min}$, and blocking in PBS with $10 \%(\mathrm{v} / \mathrm{v}) \mathrm{FBS}$ for $2 \mathrm{~h}$ at room temperature. The fixed DPSCs were then incubated with primary antibodies (1:200 dilution) specific for mouse anti- $\beta$ III-tubulin (cat. no. G712A; Promega Corporation, Madison, WI, USA) or rabbit ant-NGN2 (cat. no. sc-50401; Santa Cruz Biotechnology, Inc., Dallas, TX, USA) for $2 \mathrm{~h}$ at room temperature. Excess primary antibodies were removed by washing in $1 \%(\mathrm{w} / \mathrm{v})$ bovine serum albumin (BSA)/PBS, and the samples were incubated with the corresponding secondary antibodies (1:200 dilution), goat anti-mouse conjugated to Alexa Fluor 488 (cat. no. ab150117; Abcam, Cambridge, UK) or goat anti-rabbit conjugated to tetramethylrhodamine (TRITC; cat. no. ab6718; Abcam), for $2 \mathrm{~h}$ in the dark at room temperature. Following the removal of excess secondary antibodies by washing in $1 \%(\mathrm{w} / \mathrm{v}) \mathrm{BSA} / \mathrm{PBS}$, DAPI was used to stain the cell nuclei, and the samples were imaged under fluorescence microscopy under the specific excitation/emission wavelengths for TRITC (540/570 nm) and Alexa Fluor 488 (490/520 nm).

Statistical analysis. There were triplicate samples of cell cultures for all experimental and control groups. The data are expressed as the means \pm standard deviation, and statistically significant differences between the datasets were evaluated with Student's t-test using the SPSS 19.0 statistics software (IBM Corp., Armonk, NY, USA). The threshold for statistical significance was set at $\mathrm{P}<0.05$.

\section{Results}

Effect of EphrinB2-Fc on the neural differentiation of DPSCs. The RT-qPCR analysis of the neural markers (Fig. 1) revealed that supplementation with $2 \mu \mathrm{g} / \mathrm{ml}$ EphrinB2-Fc within the neural induction milieu resulted in significantly reduced expression of $\beta$ III-tubulin, Musashi1, NCAM, nestin, NFM and NGN2 in the DPSCs (fold expression: 0.39, 0.16, $0.40,0.38,0.06$ and 0.39 , respectively, with respect to control 


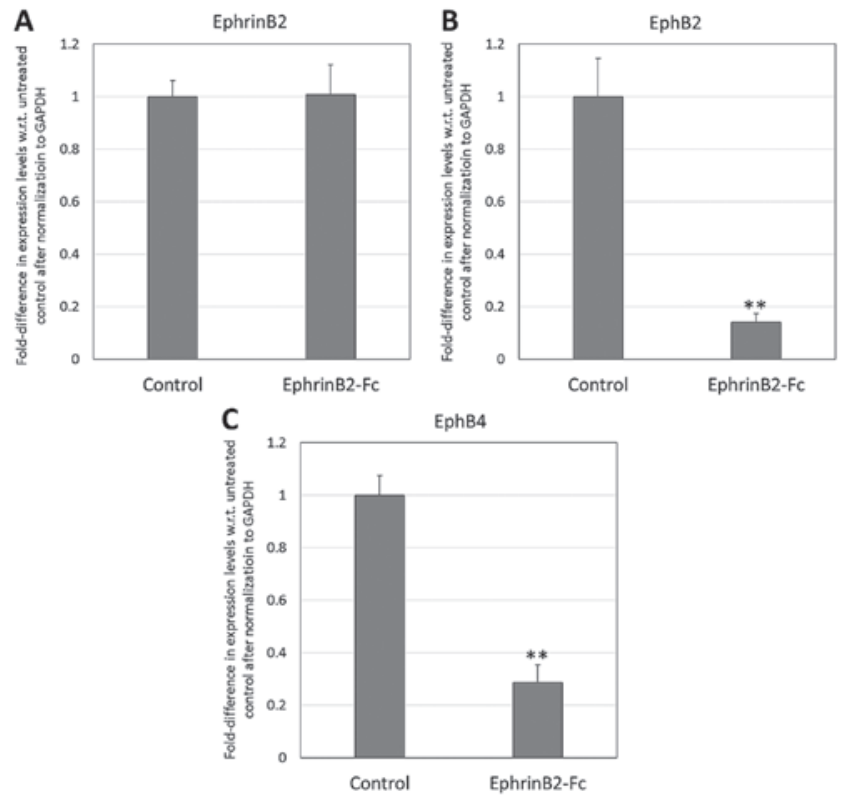

Figure 2. Reverse transcription-quantitative polymerase chain reaction analysis of (A) EphrinB2, (B) EphB2 and (C) EphB4 expression by dental pulp stem cells following treatment with $2 \mu \mathrm{g} / \mathrm{ml}$ EphrinB2-Fc in neural induction medium for 7 days. ${ }^{* *} \mathrm{P}<0.01$ vs. Control.
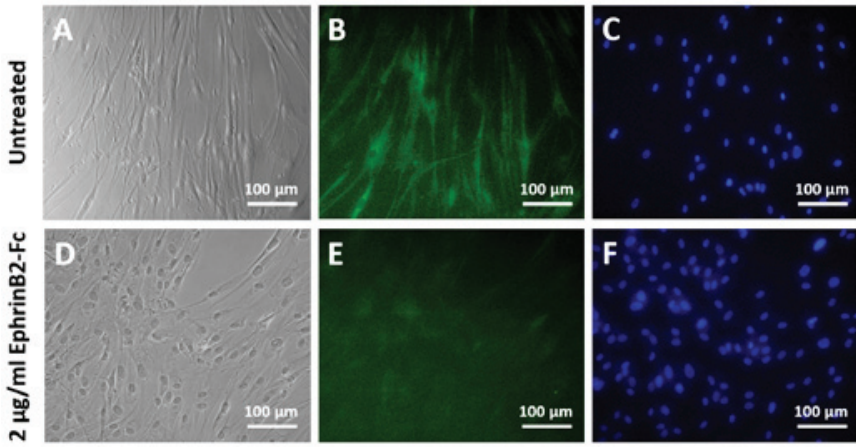

Figure 3. (A-F) Immunocytochemistry for the detection of $\beta I I I-t u b u l i n$ expression by DPSCs following treatment with $2 \mu \mathrm{g} / \mathrm{ml}$ EphrinB2-Fc in neural induction medium for 7 days. (A-C) Untreated controls. (B and E) BIII-Tubulin expression represented by green fluorescence; ( $\mathrm{C}$ and F) DAPI staining of the cell nuclei represented by blue fluorescence; (A and D) corresponding light microscopy images of the untreated and treated DPSCs, respectively. Magnification, x200. DPSC, dental pulp stem cell.

level expression in the absence of EphrinB2-Fc; $\mathrm{P}<0.01$ for Musashi1, Nestin and NFM, $\mathrm{P}<0.05$ for $\beta$ III-tubulin, NCAM and NGN2). However, there was no significant difference in the expression of MAP2 or NSE between the DPSCs EphrinB2-Fc and those without.

Additionally, as presented in Fig. 2, there was significant downregulation of EphB2 and EphB4 by the DPSCs that were exposed to EphrinB2-Fc (fold expression: 0.14 and 0.29, respectively, with respect to control level expression in the absence of EphrinB2-Fc; $\mathrm{P}<0.01)$. However, there was no significant difference in the expression of endogenous EphrinB2 by the DPSCs.

The immunocytochemistry results revealed markedly lower expression of $\beta$ III-tubulin (Fig. 3) and NGN2 (Fig. 4) by the DPSCs that were exposed to $2 \mu \mathrm{g} / \mathrm{ml}$ EphrinB2-Fc
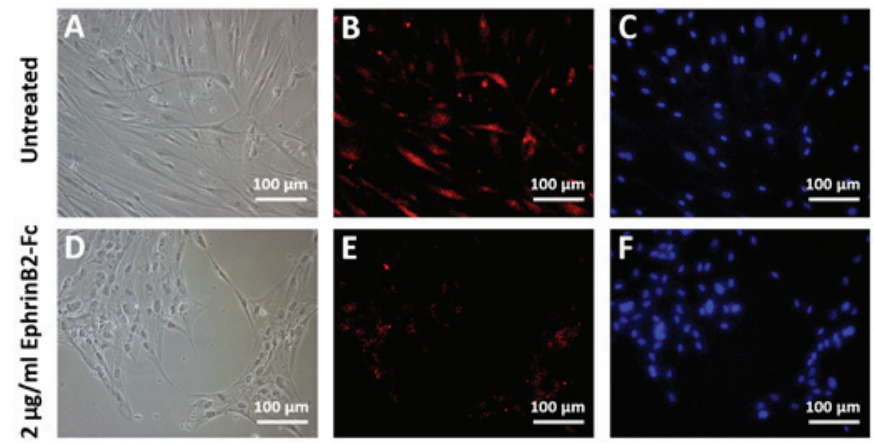

Figure 4. (A-F) Immunocytochemistry for the detection of NGN2 expression by DPSCs following treatment with $2 \mu \mathrm{g} / \mathrm{ml}$ EphrinB2-Fc in neural induction medium for 7 days. (A-C) Untreated controls; (B and E) NGN2 expression represented by red fluorescence; ( $\mathrm{C}$ and F) DAPI staining of the cell nuclei is represented by blue fluorescence; (A and D) corresponding light microscopy images of the untreated and treated DPSCs, respectively. Magnification, x200. NGN2, neurogenin 2; DPSC, dental pulp stem cell.
A

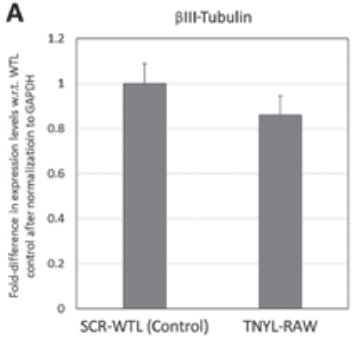

C

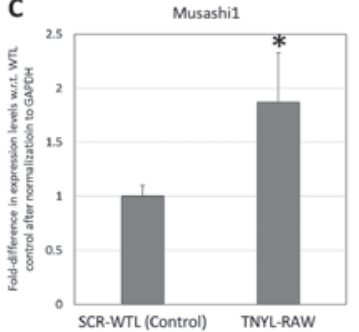

E
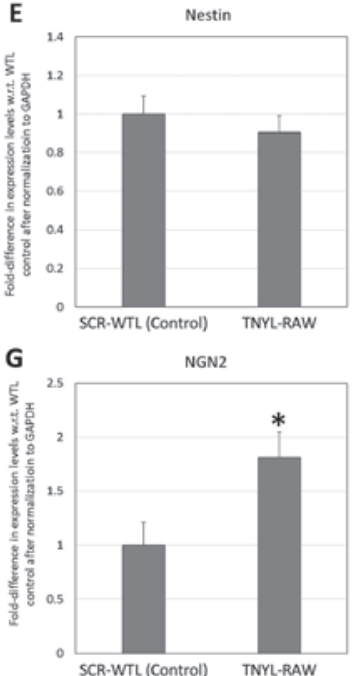

B $\quad$ MAP2

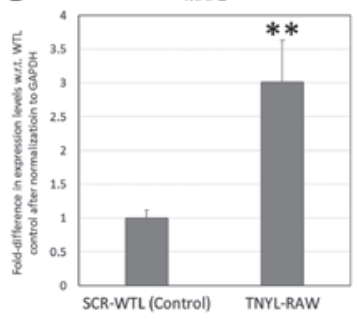

D

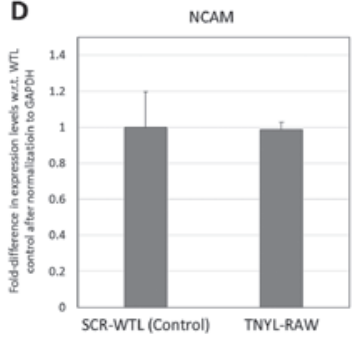

$\mathbf{F}$

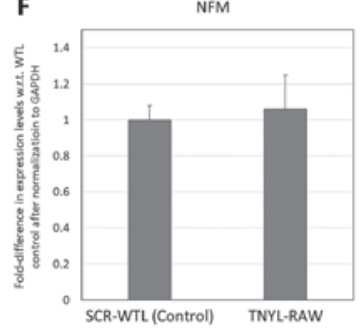

H NSE

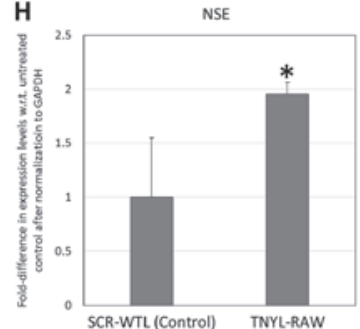

Figure 5. Reverse transcription-quantitative polymerase chain reaction analysis of neural marker expression by dental pulp stem cells following treatment with $100 \mu \mathrm{M}$ TNYL-RAW or SCR-WTL (control) peptides in neural induction medium for 7 days. Fold-difference in expression with respect to untreated controls is shown for (A) BIII-tubulin, (B) MAP2, (C) Musashil, (D) NCAM, (E) nestin, (F) NFM, (G) NGN2 and (H) NSE. ${ }^{*} \mathrm{P}<0.05$ and ${ }^{* *} \mathrm{P}<0.01$ vs. Control. MAP2, microtubule-associated protein 2 ; NCAM, neural cell adhesion molecule; NFM, neurofilament medium polypeptide; NGN2, neurogenin 2; NSE, neuron-specific enolase. 
A

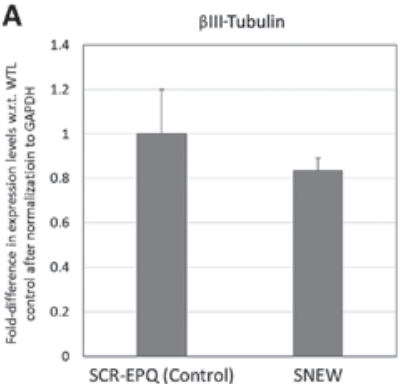

C

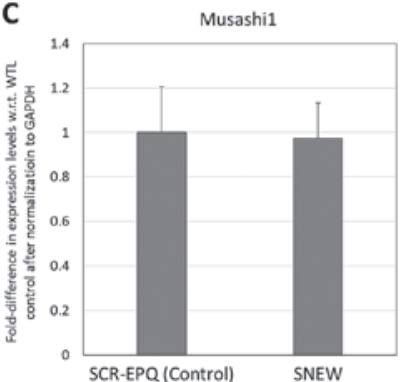

E

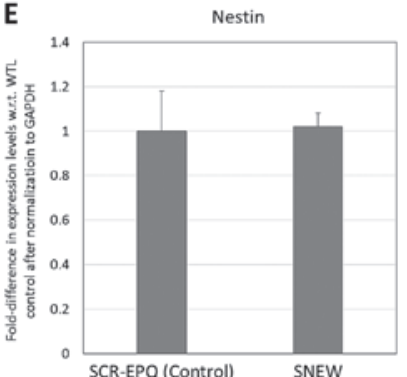

G

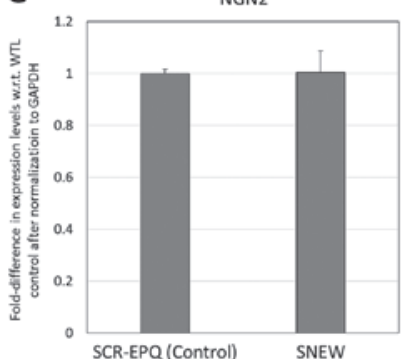

B

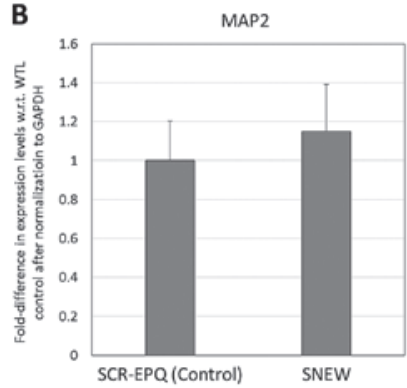

D

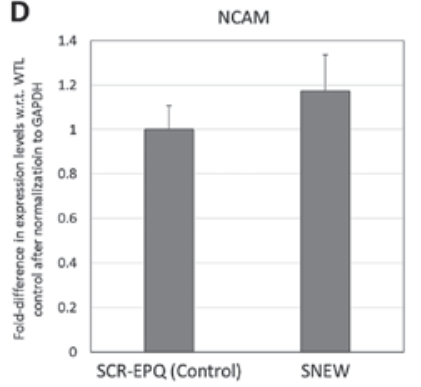

$\mathbf{F}$

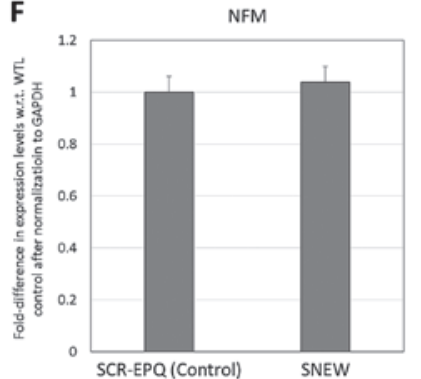

H

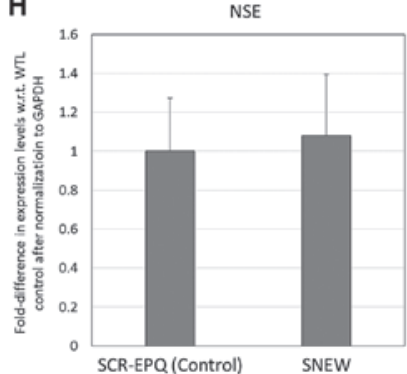

Figure 6. Reverse transcription-quantitative polymerase chain reaction analysis of neural marker expression by dental pulp stem cells following treatment with $100 \mu \mathrm{M} \mathrm{SNEW}$ or SCR-EPQ (control) peptides in neural induction medium for 7 days. Fold-difference in expression with respect to untreated controls is shown for (A) ßIII-tubulin, (B) MAP2, (C) Musashi1, (D) NCAM, (E) nestin, (F) NFM, (G) NGN2 and (H) NSE. There were no significant differences between the treated group and untreated controls ( $>>0.05)$. MAP2, microtubule-associated protein 2; NCAM, neural cell adhesion molecule; NFM, neurofilament medium polypeptide; NGN2, neurogenin 2; NSE, neuron-specific enolase.

within the neural induction milieu versus the untreated control. Additionally, treatment with EphrinB2-Fc led the DPSCs to adopt a more rounded morphology with fewer neurite outgrowths compared with the untreated controls (Figs. 3D and 4D). By contrast, the untreated DPSCs that were subjected to neural induction exhibited elongated neuron-like morphologies with obvious neurite outgrowths (Figs. 3A and 4A).

Thus, the results demonstrated that treatment with recombinant EphrinB2-Fc inhibited the neurogenic differentiation of DPSCs.
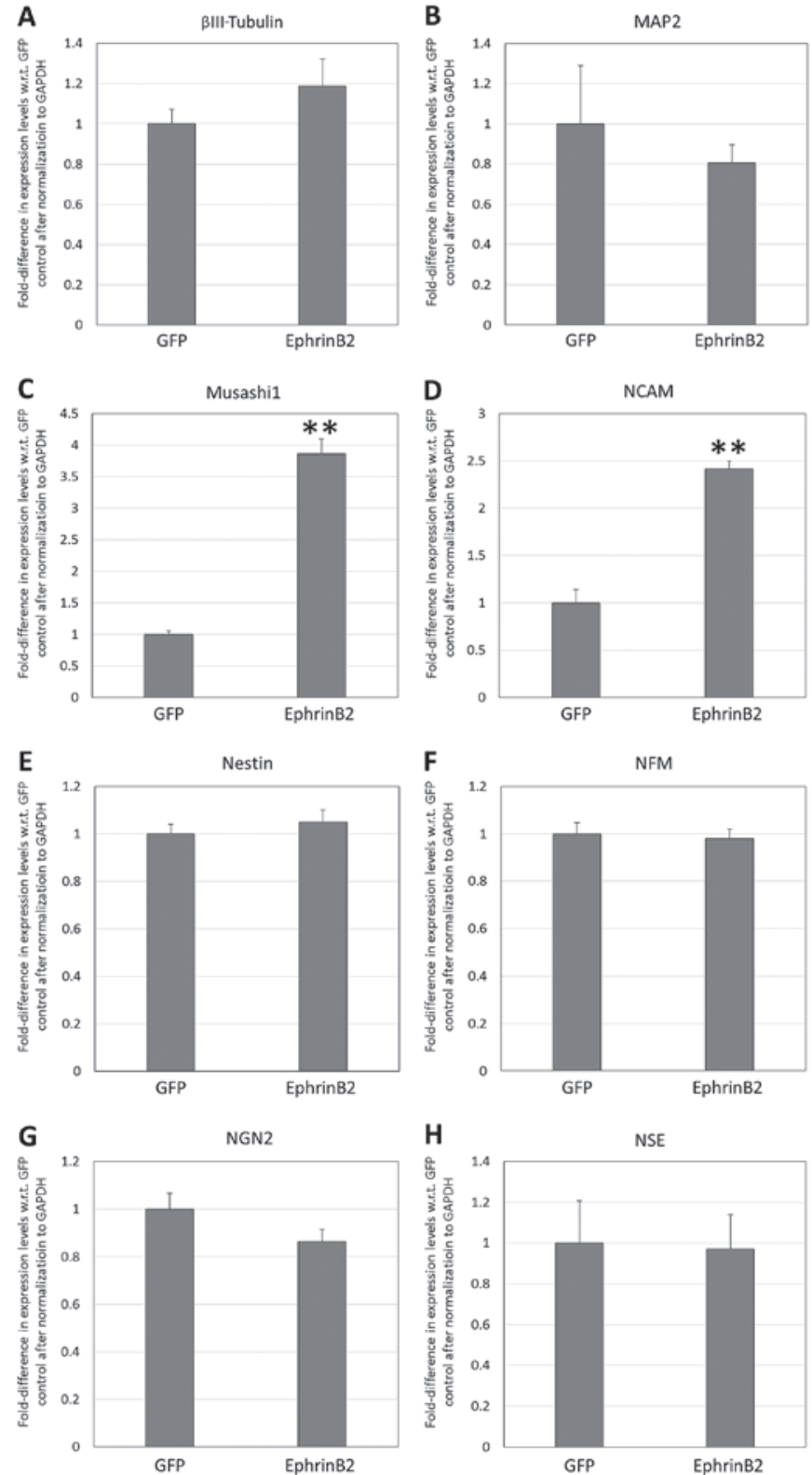

Figure 7. Reverse transcription-quantitative polymerase chain reaction analysis of neural marker expression by transgenic dental pulp stem cells overexpressing recombinant EphrinB2 following treatment with neural induction medium for 7 days. Fold-difference in expression with respect to untreated controls is shown for (A) $\beta$ III-tubulin, (B) MAP2, (C) Musashi1, (D) NCAM, (E) nestin, (F) NFM, (G) NGN2, and (H) NSE. ${ }^{* *} \mathrm{P}<0.01$ vs. Control. MAP2, microtubule-associated protein 2; NCAM, neural cell adhesion molecule; NFM, neurofilament medium polypeptide; NGN2, neurogenin 2; NSE, neuron-specific enolase.

Effects of the TNYL-RAW and SNEW peptides on the neural differentiation of DPSCs. The TNYL-RAW and SNEW peptides bind specifically to the EphB4 and EphB2 receptors, respectively, block their interactions with EphrinB2, and thus suppress forward signalling (20). The RT-qPCR analysis of the neural markers (Fig. 5) revealed that the supplementation of the neural induction milieu with 100 $\mu \mathrm{M}$ TNYL-RAW peptide resulted in significantly increased expression of MAP2, Musashi1, NGN2 and NSE by the DPSCs (fold expression: 3.01, 1.87, 1.81, and 1.95, respectively, with respect to SCR-WTL control level expression; 


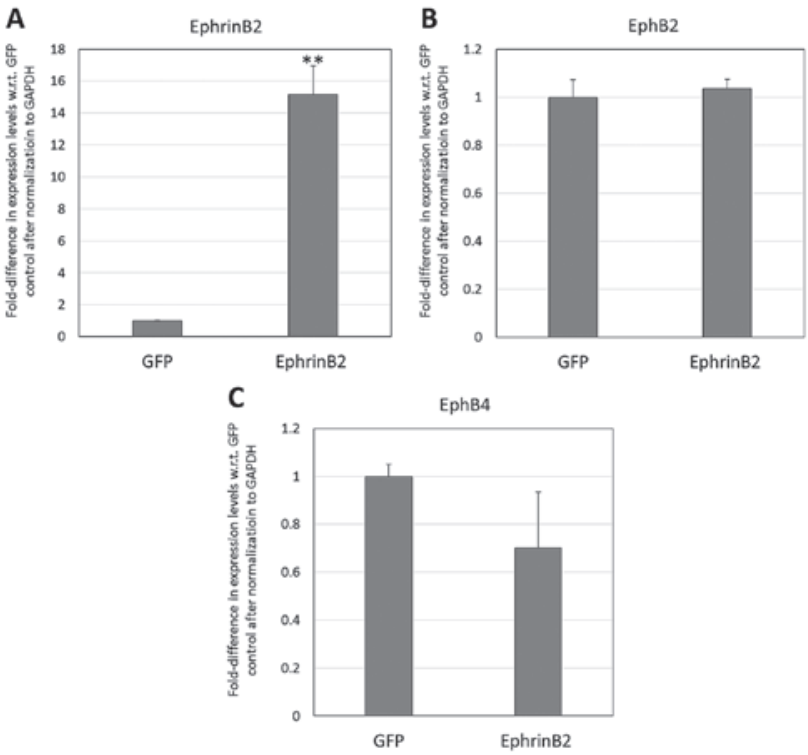

Figure 8. Reverse transcription-quantitative polymerase chain reaction analysis of (A) EphrinB2, (B) EphB2 and (C) EphB4 expression by transgenic dental pulp stem cells overexpressing recombinant EphrinB2 following treatment with neural induction medium for 7 days. ${ }^{* *} \mathrm{P}<0.01$ vs. Control. GFP, green fluorescent protein.

$\mathrm{P}<0.01$ for MAP2, $\mathrm{P}<0.05$ for Musashi1, NGN2 and NSE). By contrast, the supplementation of the neural induction milieu with $100 \mu \mathrm{M}$ SNEW peptide did not result in significant changes in any of the 8 analysed neural markers (P>0.05; Fig. 6).

Effect of transgenic expression of EphrinB2 on the neural differentiation of DPSCs. The RT-qPCR analysis of the neural markers (Fig. 7) revealed significant upregulation of Musashi1 and NCAM in the transgenic DPSCs compared with in the null-vector GFP control group (3.86- and 2.41-fold, respectively, with respect to the null-vector control levels; $\mathrm{P}<0.01$ ). The transgenic DPSCs were also identified to overexpress EphrinB2 at a level that was $15.16 \pm 1.78$-fold higher than that of the null-vector controls $(\mathrm{P}<0.01$; Fig. $8 \mathrm{~A})$, but there was no significant difference in the endogenous expression of EphB2 or EphB4 in the transgenic DPSCs versus the null-vector controls ( $\mathrm{P}>0.05$; Fig. 8B and $\mathrm{C})$.

Effect of EphB4-Fc on the neural differentiation of DPSCs. The RT-qPCR analysis of the neural markers (Fig. 9) revealed that the supplementation of the neural induction milieu with $2 \mu \mathrm{g} / \mathrm{ml}$ EphB4-Fc resulted in significant upregulation of only Musashi1 (2.00-fold with respect to the untreated control; $\mathrm{P}<0.01)$. The treatment of the DPSCs with $2 \mu \mathrm{g} / \mathrm{ml}$ EphB4-Fc did not result in any significant changes in the endogenous expression of EphrinB2, EphB2 or EphB4 (P>0.05; Fig. 10).

\section{Discussion}

Previous studies in animal models have demonstrated the role of EphrinB2 signalling in the lineage specification of motor neurons in the spinal cord (13) and dopaminergic neurons in the midbrain (15). Nevertheless, there are contradictory and conflicting data within the scientific literature regarding the role
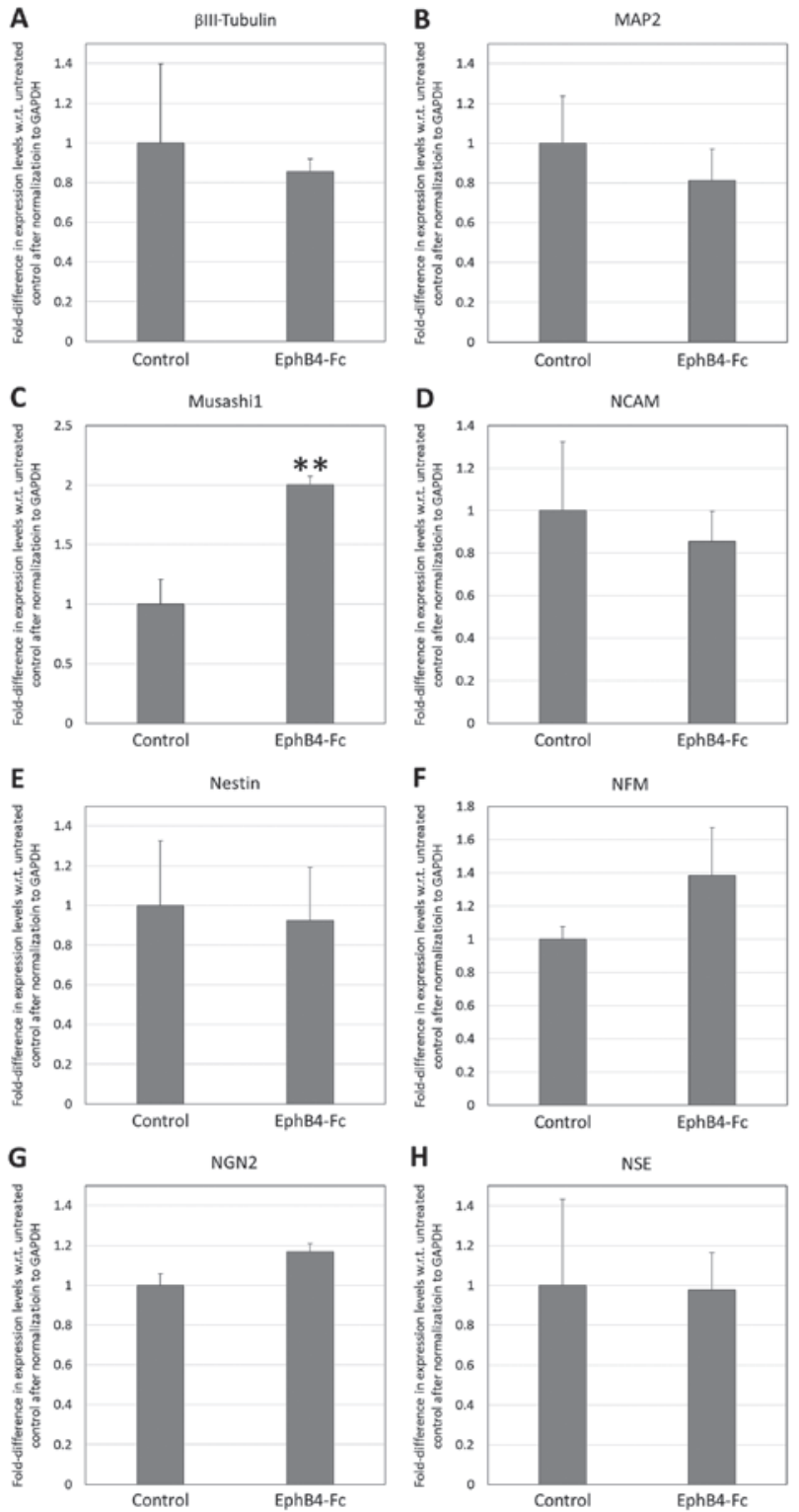

Figure 9. Reverse transcription-quantitative polymerase chain reaction analysis of neural marker expression by dental pulp stem cells following treatment with $2 \mu \mathrm{g} / \mathrm{ml}$ EphB4-Fc in neural induction medium for 7 days. Fold-difference in expression with respect to untreated controls is shown for (A) ßIII-tubulin, (B) MAP2, (C) Musashi1, (D) NCAM, (E) nestin, (F) NFM, (G) NGN2 and (H) NSE. ${ }^{* *} \mathrm{P}<0.01$ vs. Control. MAP2, microtubule-associated protein 2; NCAM, neural cell adhesion molecule; NFM, neurofilament medium polypeptide; NGN2, neurogenin 2; NSE, neuron-specific enolase.

of EphrinB2 signalling in both the promotion and inhibition of the differentiation of neural stem cells and neural progenitors. For instance, a study by Ottone et al (16) reported that EphrinB2 ligand expression by endothelial cells suppressed the differentiation of neural stem cells and thereby maintained their quiescence and 'stemness'; whereas a study by Ashton et al (17) reported that EphrinB2 expression by hippocampal astrocytes activated $\beta$-catenin, upregulated proneural transcription factors in neural stem cells, and thereby promoted neural differentiation through juxtacrine signalling. Additionally, contradictory results regarding the effects of exogenous recombinant EphrinB2 on neural differentiation have also been reported in different studies. While Xiaodong et al (26) reported that soluble recombinant EphrinB2-Fc maintained the neural progenitor cell state 


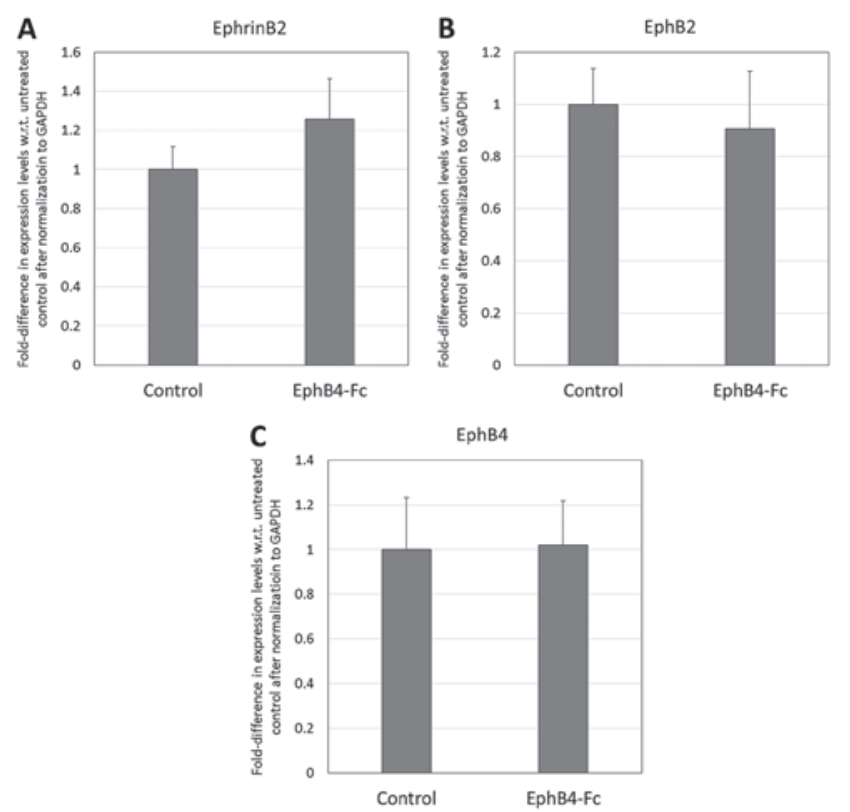

Figure 10. Reverse transcription-quantitative polymerase chain reaction analysis of (A) EphrinB2, (B) EphB2 and (C) EphB4 expression by dental pulp stem cells following treatment with $2 \mu \mathrm{g} / \mathrm{ml} \mathrm{EphB4-Fc} \mathrm{in} \mathrm{neural} \mathrm{induc-}$ tion medium for 7 days. There were no significant differences between the treated group and untreated controls $(\mathrm{P}>0.05)$.

by inhibiting differentiation, studies by Conway et al (27) and Conway and Schaffer (28) demonstrated that the recombinant ectodomain of EphrinB2 conjugated to biopolymers as multivalent ligands instead promoted the differentiation of neural stem cells.

Our results demonstrated that the treatment of DPSCs with $2 \mu \mathrm{g} / \mathrm{ml}$ EphrinB2-Fc resulted in significant inhibition of neurogenic differentiation as evidenced by RT-qPCR and immunocytochemistry results. This finding is consistent with those of Ottone et al (16) and Xiaodong et al (26), who identified that forward EphrinB2 signalling maintained the neural stem/progenitor cell state by inhibiting neurogenic differentiation. The observed rounded morphology of DPSCs after 7 days exposure to EphrinB2-Fc under neural inducing conditions, as compared with the untreated control, may be attributed to inhibition of cellular adhesion, spreading and migration by EphrinB2 signalling, as previously demonstrated by Stokowski et al (29). This involved initiation of the mitogen-activated protein kinase (MAPK) pathway by forward signalling, and phosphorylation of the Src family tyrosine kinases via reverse EphrinB signalling (29).

It may be hypothesized that the observed downregulation of EphB2 and EphB4 in the DPSCs treated with EphrinB2-Fc may be due to a negative feedback inhibition loop, triggered in response to excessive EphrinB2-Fc binding that activates EphB2 and EphB4 receptors on DPSCs. Previously, Kawano et al (30) reported that overstimulation with high dosages of EphrinB2-Fc could trigger a negative feedback mechanism in the context of T-cell proliferation. It is possible that the downregulation of EphB2 and EphB4 may form a part of this negative feedback mechanism, though this requires further investigation.

The observed inhibitory effects of EphrinB2 forward signalling on the neurogenic differentiation of DPSCs prompted investigation into whether specific peptide inhibitors of EphrinB2-EphB4 and EphrinB2-EphB2 signalling could instead promote the neurogenic differentiation of DPSCs. The TNYL-RAW and SNEW peptides, which specifically inhibit the EphB4 and EphB2 receptors, respectively (21), together with the corresponding control scrambled peptides (SCR-WTL and SCR-EPQ, respectively) were utilized. The results indicated that the TNYL-RAW peptide (specific for EphB4) could enhance neurogenic differentiation as evidenced by the upregulation of 4 neural markers (MAP2, Musashi1, NGN2 and NSE). By contrast, the SNEW peptide (specific for EphB2) did not elicit significant changes in the expression of any of the 8 analysed neural markers. Thus, it may be deduced that the EphrinB2-EphB4 interaction serves a more prominent role in the neurogenesis of DPSCs relative to the EphrinB2-EphB2 signalling axis.

Subsequently, the present study investigated the effects of transgenic overexpression of EphrinB2 on the neural differentiation of DPSCs. The overexpressed EphrinB2 may stimulate both forward and reverse signalling in the transgenic DPSCs. The current results demonstrated that there was only a slight enhancement of neural differentiation in the transgenic DPSCs overexpressing EphrinB2, and only 2 neural markers (Musashil and NCAM) were upregulated. As the results on EphrinB2-Fc indicated that forward signalling inhibited neurogenic differentiation in DPSCs, the marginal enhancement of neurogenesis may possibly arise from an increase in reverse signalling, mediated via activation of the overexpressed EphrinB2 by either endogenous EphB2 or EphB4 receptors on adjacent cells. This notion in turn prompted investigation into whether the treatment of DPSCs with soluble recombinant EphB4 to stimulate reverse signalling could enhance the neurogenic differentiation of DPSCs. However, the results revealed a significant increase in the expression of the neural marker Musashil only.

Therefore, to conclude, stimulation of forward EphrinB2-EphB4 signalling markedly inhibited the neurogenesis of DPSCs, whereas suppression of this forward signalling pathway with the TNYL-RAW peptide inhibitor promoted neural differentiation. Additionally, stimulation of reverse EphB4-EphrinB2 signalling was also observed to marginally enhance the neural differentiation of DPSCs. Future studies by our group will investigate whether combining the TNYL-RAW peptide with inhibitors of other signalling pathways can produce a synergistic effect on the promotion of DPSC neurogenesis. Possible candidates include inhibitors of the Jagged-Notch signalling pathway, since this particular signalling pathway was demonstrated in a previous study (16) to work together with forward EphrinB2 signalling to inhibit the differentiation of neural stem cells. Such peptide or small molecule inhibitors of EphrinB2 forward signalling may have potential clinical applications in therapies based on neural tissue engineering with DPSCs.

\section{Acknowledgements}

The authors would like to thank Ms. Edith Tong of the Faculty of Dentistry of the University of Hong Kong for her assistance in performing the various experiments of the present study. 


\section{Funding}

The present study was supported by a grant from the National Nature Science Foundation of China (grant no. 81470735) and a General Research Fund (GRF) grant from the Research Grants Council of Hong Kong (grant no. HKU17126914) to CZ.

\section{Availability of data and materials}

The datasets used and/or analyzed during the current study are available from the corresponding author on reasonable request.

\section{Authors' contributions}

$\mathrm{BCH}$ performed the experiments, analyzed and interpreted the data and wrote the draft manuscript. TG, JX and LWL aided with the experiments and data collection and critically edited the manuscript. CZ designed and managed the study. All authors have read and approved the final version of the manuscript.

\section{Ethics approval and consent to participate}

The research protocol was reviewed and approved by the Human Research Ethics Committee of the University of Hong Kong, China. All subjects provided written informed consent for participation in the study.

\section{Consent for publication}

All subjects provided written informed consent permitting the publication of relevant data following anonymization of personally identifiable information.

\section{Competing interests}

All authors declare that they have no competing interests.

\section{References}

1. Gronthos S, Mankani M, Brahim J, Robey PG and Shi S: Postnatal human dental pulp stem cells (DPSCs) in vitro and in vivo. Proc Natl Acad Sci USA 97: 13625-13630, 2000.

2. Gronthos S, Brahim J, Li W, Fisher LW, Cherman N, Boyde A, DenBesten P, Robey PG and Shi S: Stem cell properties of human dental pulp stem cells. J Dent Res 81: 531-535, 2002.

3. Huang GT, Gronthos S and Shi S: Mesenchymal stem cells derived from dental tissues vs. those from other sources: Their biology and role in regenerative medicine. J Dent Res 88: 792-806, 2009.

4. Nuti N, Corallo C, Chan BM, Ferrari M and Gerami-Naini B: Multipotent differentiation of Human dental pulp stem cells: A literature review. Stem Cell Rev 12: 511-523, 2016

5. Sharpe PT: Dental mesenchymal stem cells. Development 143 2273-2280, 2016.

6. Heng BC, Lim LW, Wu W and Zhang C: An overview of protocols for the neural induction of dental and oral stem cells in vitro. Tissue Eng Part B Rev 22: 220-250, 2016.

7. Chai Y, Jiang X, Ito Y, Bringas P Jr, Han J, Rowitch DH, Soriano P, McMahon AP and Sucov HM: Fate of the mammalian cranial neural crest during tooth and mandibular morphogenesis. Development 127: 1671-1679, 2000.

8. Song M, Lee JH, Bae J, Bu Y and Kim EC: Human dental pulp stem cells are more effective than human bone marrow-derived mesenchymal stem cells in cerebral ischemic injury. Cell Transplant 26: 1001-1016, 2017.
9. Yang C, Li X, Sun L, Guo W and Tian W: Potential of human dental stem cells in repairing the complete transection of rat spinal cord. J Neural Eng 14: 026005, 2017.

10. Sasaki R, Aoki S, Yamato M, Uchiyama H, Wada K, Ogiuchi H, Okano T and Ando T: PLGA artificial nerve conduits with dental pulp cells promote facial nerve regeneration. J Tissue Eng Regen Med 5: 823-830, 2011.

11. Mead B, Logan A, Berry M, Leadbeater W and Scheven BA: Intravitreally transplanted dental pulp stem cells promote neuroprotection and axon regeneration of retinal ganglion cells after optic nerve injury. Invest Ophthalmol Vis Sci 54: 7544-7556, 2013.

12. Arthur A, Koblar S, Shi S and Gronthos S: Eph/ephrinB mediate dental pulp stem cell mobilization and function. J Dent Res 88: 829-834, 2009.

13. Laussu J, Audouard C, Kischel A, Assis-Nascimento P, Escalas N, Liebl DJ, Soula C and Davy A: Eph/Ephrin signaling controls progenitor identities in the ventral spinal cord. Neural Dev 12: 10, 2017.

14. Luxey M, Laussu J and Davy A: EphrinB2 sharpens lateral motor column division in the developing spinal cord. Neural Dev 10: 25, 2015 .

15. Yue Y, Widmer DA, Halladay AK, Cerretti DP, Wagner GC, Dreyer JL and Zhou R: Specification of distinct dopaminergic neural pathways: Roles of the Eph family receptor EphB1 and ligand ephrin-B2. J Neurosci 19: 2090-2101, 1999.

16. Ottone C, Krusche B, Whitby A, Clements M, Quadrato G, Pitulescu ME, Adams RH and Parrinello S: Direct cell-cell contact with the vascular niche maintains quiescent neural stem cells. Nat Cell Biol 16: 1045-1056, 2014.

17. Ashton RS, Conway A, Pangarkar C, Bergen J, Lim KI, Shah P, Bissell M and Schaffer DV: Astrocytes regulate adult hippocampal neurogenesis through ephrin-B signaling. Nat Neurosci 15: 1399-1406, 2012.

18. Barquilla A and Pasquale EB: Eph receptors and ephrins: Therapeutic opportunities. Annu Rev Pharmacol Toxicol 55: 465-487, 2015.

19. Nikolov DB, Xu K and Himanen JP: Eph/ephrin recognition and the role of Eph/ephrin clusters in signaling initiation. Biochim Biophys Acta 1834: 2160-2165, 2013.

20. Heng BC, Wang S, Gong T, Xu J, Yuan C and Zhang C: EphrinB2 signaling enhances osteogenic/odontogenic differentiation of human dental pulp stem cells. Arch Oral Biol 87: 62-71, 2018.

21. Chrencik JE, Brooun A, Recht MI, Nicola G, Davis LK, Abagyan R, Widmer H, Pasquale EB and Kuhn P: Three-dimensional structure of the EphB2 receptor in complex with an antagonistic peptide reveals a novel mode of inhibition. J Biol Chem 282: 36505-36513, 2007.

22. Zou T, Dissanayaka WL, Jiang S, Wang S, Heng BC, Huang X and Zhang C: Semaphorin 4D enhances angiogenic potential and suppresses osteo-/odontogenic differentiation of human dental pulp stem cells. J Endod 43: 297-305, 2017.

23. Heng BC, Gong T, Wang S, Lim LW, Wu W and Zhang C: Decellularized matrix derived from neural differentiation of embryonic stem cells enhances the neurogenic potential of dental follicle stem cells. J Endod 43: 409-416, 2017.

24. Davis S, Gale NW, Aldrich TH, Maisonpierre PC, Lhotak V, Pawson T, Goldfarb M and Yancopoulos GD: Ligands for EPH-related receptor tyrosine kinases that require membrane attachment or clustering for activity. Science 266: 816-819, 1994.

25. Livak KJ and Schmittgen TD: Analysis of relative gene expression data using real-time quantitative PCR and the 2(-Delta Delta C(T)) Method. Methods 25: 402-408, 2001.

26. Xiaodong H, Zhen H, Min S, Zhiming C, Hongyan J, Chong Z, Xuefeng $\mathrm{T}$ and Guohua J: Direct inhibition of cell surface ephrin-B2 by recombinant ephrin-B2/FC. Biochem Biophys Res Commun 440: 300-305, 2013.

27. Conway A, Vazin T, Spelke DP, Rode NA, Healy KE, Kane RS and Schaffer DV: Multivalent ligands control stem cell behaviour in vitro and in vivo. Nat Nanotechnol 8: 831-838, 2013.

28. Conway A and Schaffer DV: Biomaterial microenvironments to support the generation of new neurons in the adult brain. Stem Cells 32: 1220-1229, 2014.

29. Stokowski A, Shi S, Sun T, Bartold PM, Koblar SA and Gronthos S: EphB/ephrin-B interaction mediates adult stem cell attachment, spreading, and migration: Implications for dental tissue repair. Stem Cells 25: 156-164, 2007.

30. Kawano H, Katayama Y, Minagawa K, Shimoyama M, Henkemeyer $M$ and Matsui T: A novel feedback mechanism by Ephrin-B1/B2 in T-cell activation involves a concentration-dependent switch from costimulation to inhibition. Eur J Immunol 42: 1562-1572, 2012. 